\title{
Flight Test Measurement and Assessment of a Flapping Micro Air Ve- hicle
}

\author{
Jong Heon Kim*, Chan Yik Park ${ }^{* *}$ and Seungmoon Jun** \\ Aeronautical Tech. Directorate, Agency for Defense Development, Daejeon, Korea
}

\author{
Dae Keun Chung***, Jong Rok Kim ${ }^{* * *}$ and Hee Chul Hwang*** \\ Dept. of PGM Technology, Hanwha Corp. R\&D Center, Daejeon, Korea
}

\author{
Bret Stanford $^{* * * *}$, Philip Beran ${ }^{* * *}$, Gregory Parker ${ }^{* * * *}$ and Denny Mrozinski ${ }^{* * * *}$
}

Air Vehicles Directorate, Air Force Research Laboratory, WPAFB, OH, USA

\begin{abstract}
Flight test of flapping micro air vehicles (FMAVs) is carried out using an instrumented measurement system to obtain various engineering parameters and hence to assess the flight performance of the vehicles through the data investigation. An indoor flight test facility equipped with a motion capture system and tracking cameras is used for the work presented in this paper. Maneuvers including straight-level flight, ground flapping, takeoff and landing are tested. Spatial position and orientation data are obtained from the retro-reflective tracking markers attached to the vehicles. Subsequent test analysis is carried out by generating performance parameters from raw data and then assessing the flight performance by comparison of the vehicles. The main findings of this work confirm that the test method and procedures presented here enable the systematic numerical data measurement and assessment of the flying performances of these vehicles, and show the applicability for the test and evaluation of general flapping MAVs.
\end{abstract}

Key words: Micro Air Vehicle (MAV), Flapping Wing, Motion Capture Tracking System, Flight Test and Assessment

\section{Introduction}

A number of different types of MAVs, inspired by natural flyers such as birds and insects, have been developed for surveillance and reconnaissance missions, and for their affordable development cost compared to conventional aircraft. In particular, MAVs with flapping wings (noted as FMAV hereafter) are expected to have high maneuverability with the advantages of low-power requirements and high aerodynamic efficiency, and as such, many diverse experimental and numerical studies have appeared in the literature. However, the design and manufacturing of FMAVs rely strongly upon trial and error, as well as the empirical design skills of the developers, because the complicated flight mechanics and principle of flapping flyers have not yet fully understood for the systematic development of FMAVs. The test and evaluation of FMAVs are likewise immature, so performance assessment is mostly done by visual observation
This is an Open Access article distributed under the terms of the Creative Commons Attribution Non-Commercial License (http://creativecommons.org/licenses/bync/3.0// which permits unrestricted non-commercial use, distribution, and reproduction in any medium, provided the original work is properly cited.

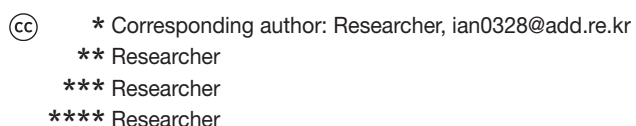


of vehicle flight and by subjective judgment.

Past studies regardingFMAVsinvestigated the flow patterns and vortex structures of the flapping wings, to examine the basic flight principles [1]. Another study tried to calculate the aerodynamic resultants acting on the wings, based on an investigation of the unsteady airflow characteristics [2]. However, these studies were not connected to an actual aerial flight test of the system. Instead, most of these tests were conducted in a wind tunnel with the vehicle at a fixed position, and therefore, the real characteristics of vehicle flight were not correctly represented. The reason that many FMAV tests are limited to visual observation, pilot feedback, or wind tunnel testing is that conventional aircraft test methods using many gages and wires are not dimensionally compatible with FMAVs. Consequently, this study pursues a different testing approach that will provide a more comprehensive understanding of a FMAV in flight.

In this study, a test to obtain flight characteristics of a FMAV is performed by using a flight test measurement system. This paper presents the procedure and results of the instrumented flight test that was performed on the vehicles we developed. The test was performed in the indoor flight test facility at the Air Force Research Laboratory (AFRL) of Wright-Patterson AFB, equipped with a Vicon motion capture system [3] and tracking cameras. Real-time spatial position and orientation data were obtained from the small retro-reflective tracking markers attached to the vehicles. Along with voltage, current, time and other raw data from the transmitter, the test results were analyzed to generate flight parameters and hence to assess the performance of each vehicle.

Motion capture systems were originally developed for use in areas such as film production, ergonomics, etc. This approach was applied to MAV testing recently in some university laboratories $[4,5]$, but the scope of these tests was limited to control identification of rotary MAVs because of the limited facility size. The test described in this study is a full-scale FMAV flight test, supported by a dedicated facility with enough space and equipment to cover a large range of dynamic behavior of flapping vehicles, applying a relevant approach for the identification and assessment of the performance characteristics of the vehicles.

\section{Test Details}

\subsection{Test Facility and Equipment}

Conventional flight or ground test of an aircraft is usually accompanied with numerous sensors or gages connected to on-board data acquisition devices. However, as this approach is virtually inapplicable to MAVs due to their small dimensions and stringent weight requirements (as mentioned in the Introduction), a completely different approach is required for the test and evaluation of MAVs.

The flight test for this study was conducted in the MAV indoor flight test facility at AFRL as shown in Fig. 1 . The test chamber is $24 \mathrm{~m} \times 15 \mathrm{~m}$ wide, equipped with a visual motion capture system (called Vicon system hereafter) with 60 cameras capable of real-time vehicle flight data acquisition from the attached markers on a vehicle. The spherical markers are available in various sizes, but most of them are very small and light with no need for wires. Therefore, they are non-intrusive on the MAV airframe.

The brief qualitative description of the system used here is illustrated in Fig. 2 and Fig. 3. When the tracking cameras detect the reflective markers on the vehicle at each time frame, the Vicon system correlates the camera data with the vehicle's position and orientation. Data were collected at $200 \mathrm{~Hz}$ with an estimated position error of $\pm 0.2 \mathrm{~mm}$. And the data were converted into ASCII format and transferred to a dedicated computer for post-analysis.

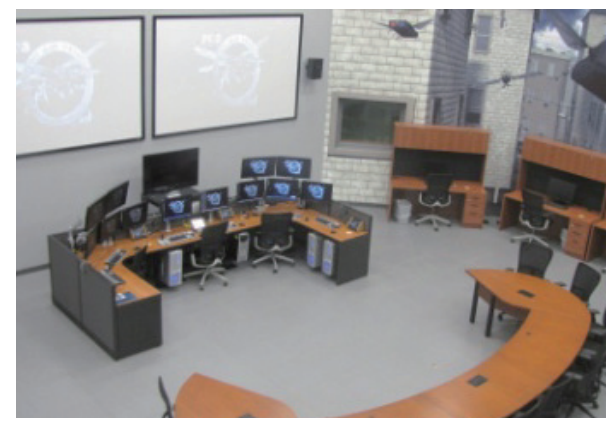

(a) Control Room

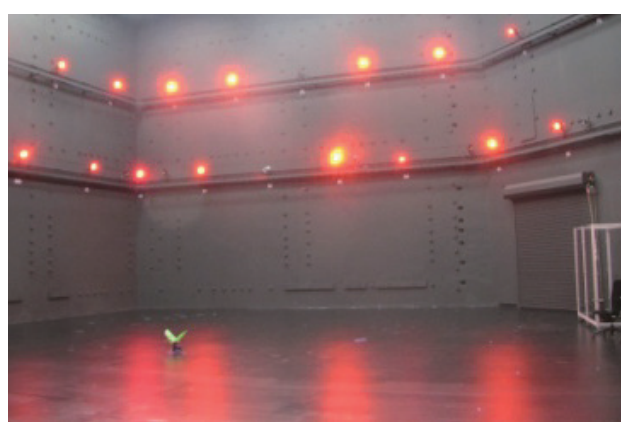

(b) Vehicle in the facility with cameras

Fig. 1. MAV Indoor Flight Test Facility 


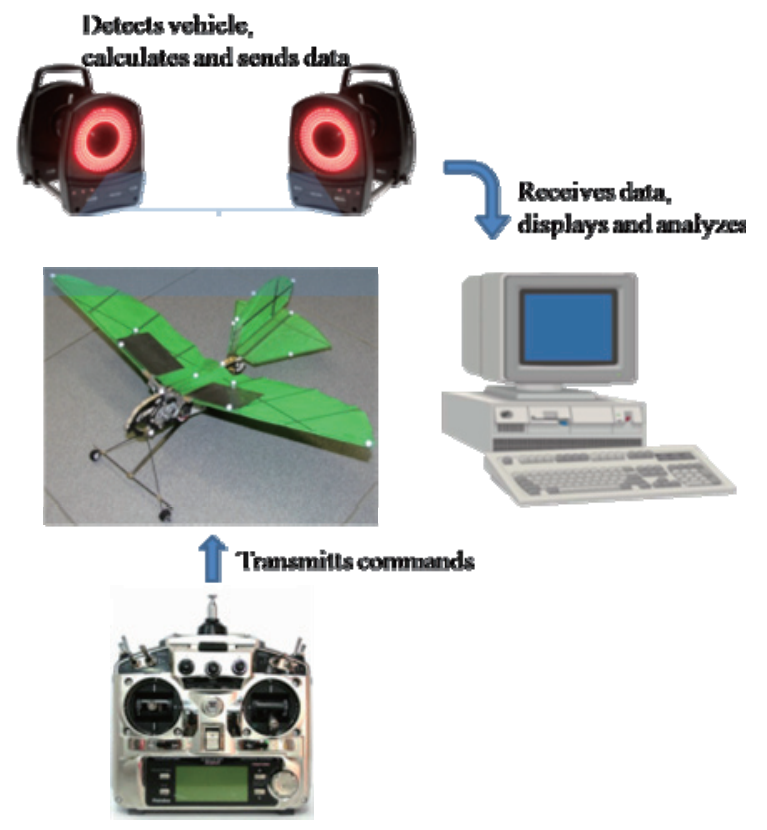

Fig. 2. Schematic of Flight Test Measurement Procedure
Since the motion capture system was originally developed for relatively slow motions such as those of the human body, even with the state-of-the-art system used here, the rapid dynamics of the wing motion of a FMAV is beyond the tracking capacity of the system. For this reason some data loss or signal spike is inevitable, especially when the wing is highly flexible. Data selection and interpretation, along with a good understanding of the limit of the test equipment, become critical.

\subsection{Test Preparation and Execution}

Three flapping MAVs were tested as shown from Fig. 4 to Fig. 6, each with a wing span of $50 \mathrm{~cm}$. The configurations of vehicle_1 and vehicle_2 are differentiated by their wing driving mechanisms: vehicle_l has a single driving crank whereas vehicle_2 has a double crank. In the single crank mechanism of vehicle_1, the crank and its connecting rod are linked to the wing frame, and the rotation of the crank produces the wing flapping motion accordingly. As the single crank drives both of the front and rear spars, the wing twisting angle during flapping is determined by the airflow and spar stiffness. In the double crank mechanism of vehicle_2 on the

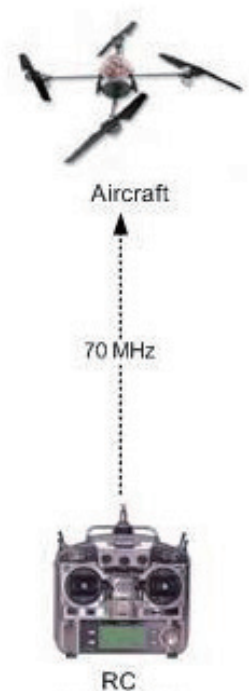

Transmitter

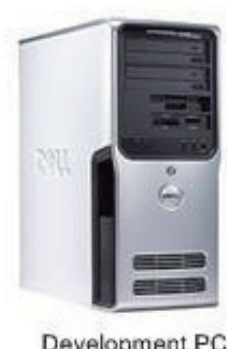

Development PC

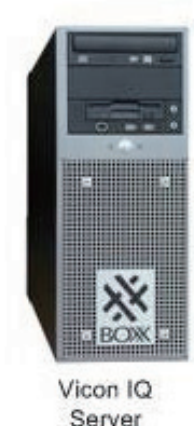

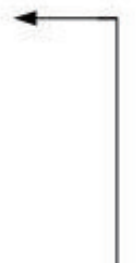

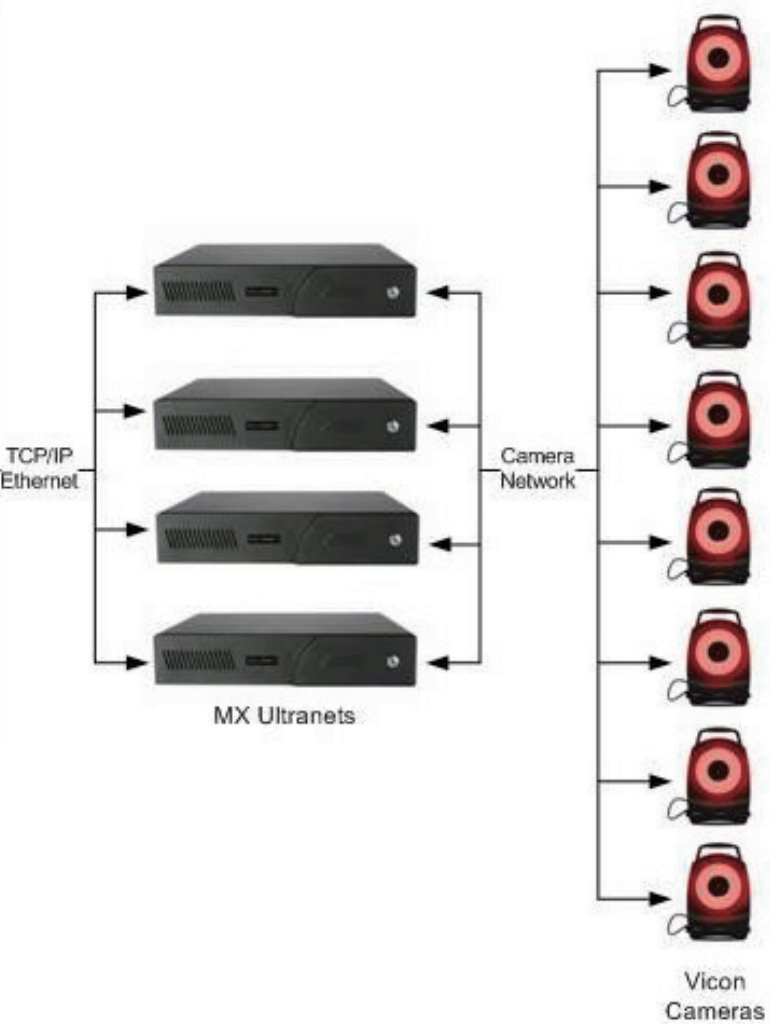

Fig. 3. Schematic of Visual Tracking System 

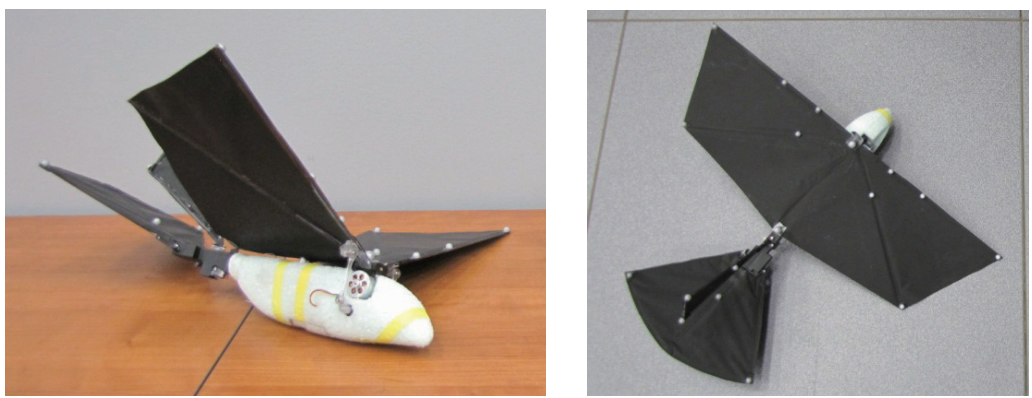

Fig. 4. Vehicle_1 with Markers
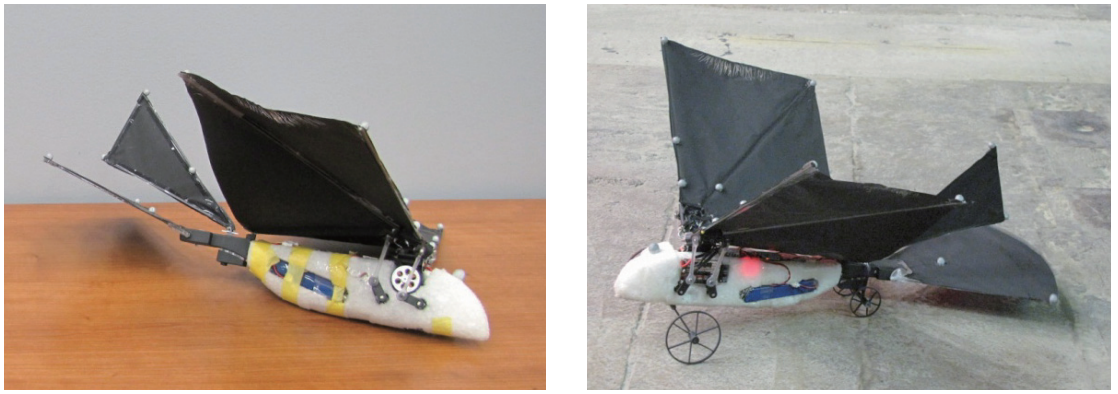

Fig. 5.Vehicle_2 with Markers
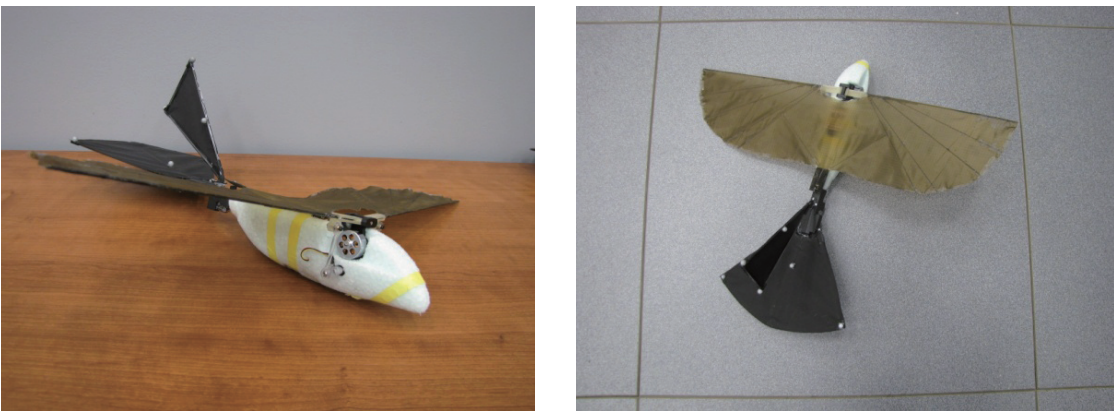

Fig. 6. Vehicle_3 with Markers

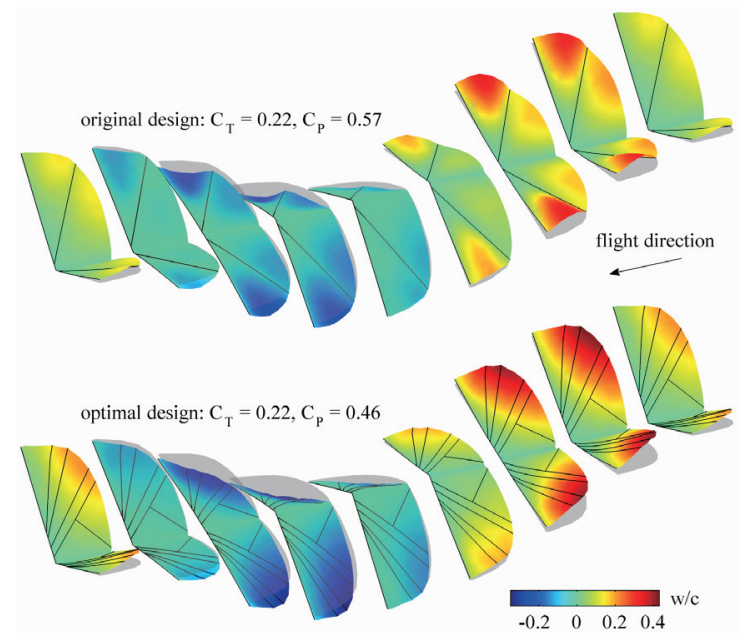

Fig. 7. Aeroelastic Simulation Result of Vehicle_3 Wing [7] other hand, the front crank and its connecting rod are linked to the front spar through the wing frame while the rear crank and its connecting rod are attached to the rear spar. The phase angle existing between the two cranks generates wing twisting during the vehicle's flapping. Because this phase angle is a design parameter, wing twisting can be actively controlled to enhance the flight performance. The double crank mechanism was developed to increase the twisting during wing flapping. Because the increased twisting or the flexibility of the wing in a FMAV produces stronger wing tip vortices and hence contribute to higher power and flapping efficiency [6], the double crank mechanism or the active wing twisting structure was expected to accommodate the heavier vehicle_2, which was loaded with subsystems and a payload. The takeoff weight of vehicle_2 was $210 \mathrm{~g}$, while that of vehicle_1 was $130 \mathrm{~g}$. 
Vehicle_3 has the same configuration as vehicle_1 except for the structural layout of the main wing. The main wing of vehicle_3 was designed by aeroelastic topology optimization to find the optimal wing batten structure, in order to minimize the required power consumption under the constraint of no decrease in aerodynamic thrust. The resulting wing structure, as shown in Fig. 6, has considerably more battens than the wings discussed above, each of which is also thinner. This design results in a relatively smooth wing deformation pattern throughout a stroke (refer to the simulation results of Fig. 7)[7]. The takeoff weight of vehicle_3 was 120g, slightly lighter than vehicle_1, to make the vehicle with the relatively smaller wing flyable.

Since the flight was manually controlled, more than 10
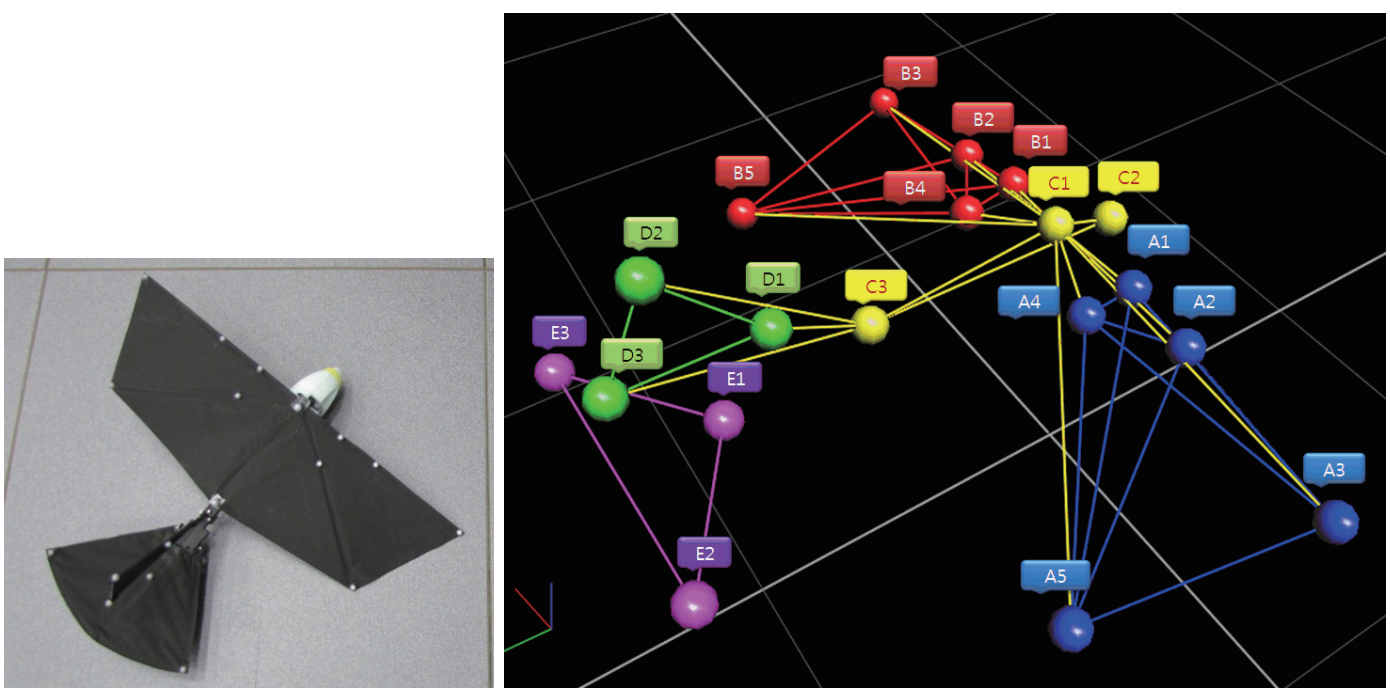

Fig. 8. Markers Labeling of Vehicle in Vicon Display

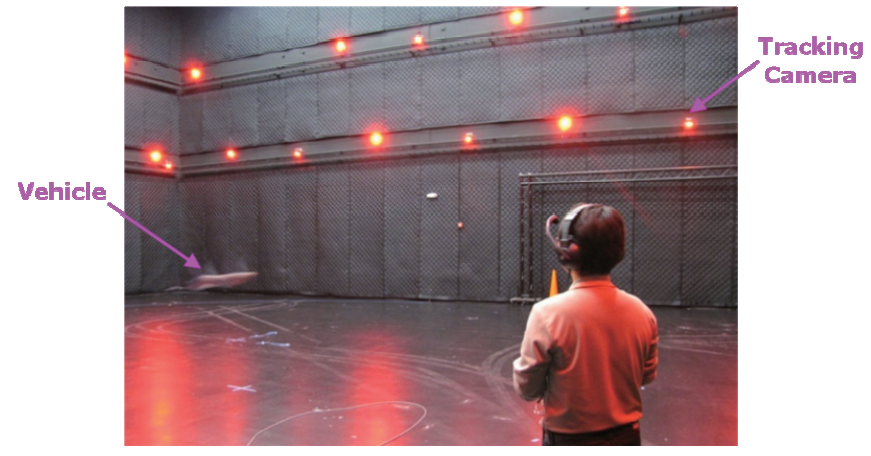

(a) Level Flight Test

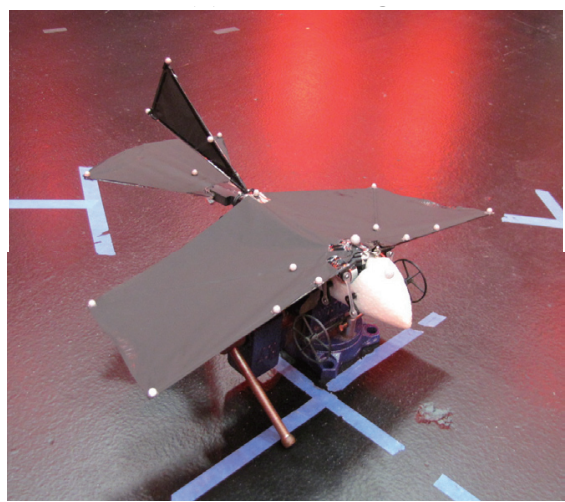

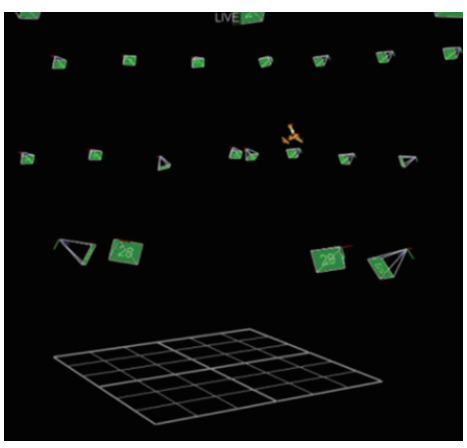

(b) Real-Time Display in Tracking System

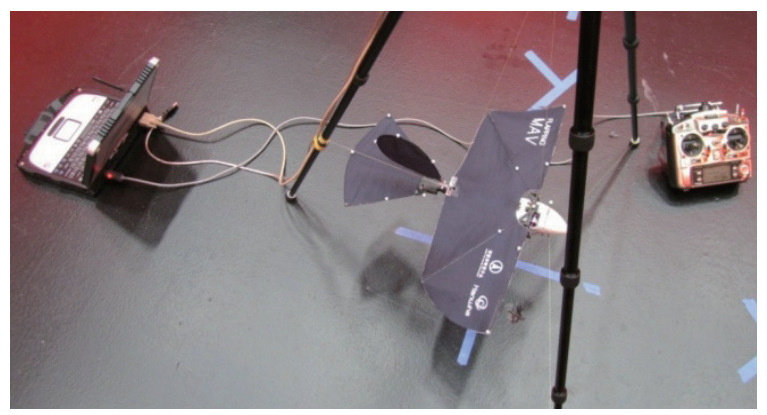

(c) Ground Flapping Test

Fig. 9. Vehicle Test in Test Chamber 
flights for each vehicle were made to counterbalance the inherent deviations and to obtain the most representative result. Flight maneuvers included ground flapping and straight-level flight, and takeoff/landing. The ground flapping was tested to calibrate and validate the test data of the system, and the takeoff/landing was used to assess the vehicle's takeoff/landing capability.

The prepared vehicles with markers for the test, and the corresponding vehicle model setup in the system display are shown in Fig. 8. The test system recognizes a vehicle as a skeleton structure made of tracking markers linked to each other, and detects them during a flight at a designated sampling rate. Some pictures taken during the test are shown in Fig. 9.

\subsection{Test Data Investigation and Assessment}

The raw data obtained from the tracking system consist of the real-time spatial position and orientation of a vehicle. Through proper filtering, selection, application of derivatives or editing of data to calculate parameters such as velocity or acceleration, the flight performance of a vehicle can be assessed.

First, the Vicon tracking data were validated by several methods, including the comparison between the flapping frequency measured from tracking data and that measured from the captured video data, obtained from the normal cameras installed on the wall of the test chamber. The trajectory and velocity data of straight and circular level flights were also used for comparison.

Fig. 10 illustrates a selected validation case, the height data of the wing and fuselage in a straight-level flight, which was used to determine if the system could reproduce the realistic typical motion of FMAVs. The data with the larger height amplitude indicates the z-position data of the right wing tip marker (A3 in Fig. 8) and the data with the smaller height amplitude indicates the z-position data of the front body marker (C2 in Fig 8). It was discovered from the investigation that maintaining a fixed altitude was not feasible because slight body fluctuations were inevitable due to the periodic beating of the wing. However, since this 'intrinsic' characteristic of FMAVs is also found in nature such as in the flight of birds, it is more realistic to incorporate oscillatory altitude data in straight level flight analysis [8]. Consequently, it is reasonable to set the straight level standard of FMAVs not on a numerical reference, such as that of a conventional fixed wing aircraft, but on a realistic reference as found in nature. This is one of the many examples which address the need to create test standards specific to FMAVs in future research.

Table 1 summarizes the performance parameters we identified for the practical assessment and comparison of FMAVs. Most of the parameters were derived from raw data - position and orientation data - through mathematical calculation. For example, velocity data were obtained through a finite difference method using position vs. time data. Acceleration data were obtained from velocity data likewise. Angle of attack (AoA) was the angle calculated between the velocity vector and forward body axis, and flapping frequency was obtained by calculating the time interval between two points of inflection in the wing altitude data plot (seen in Fig. 10, for example). In addition, the

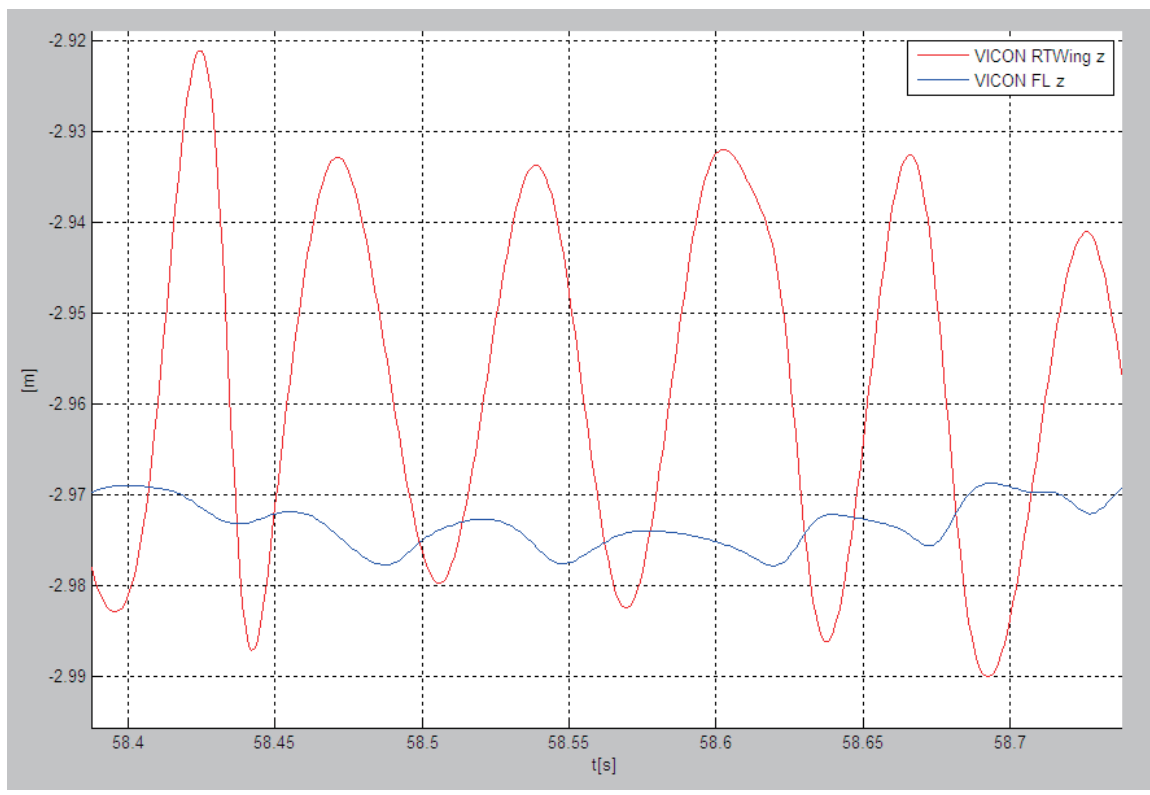

Fig. 10. Height vs. Time Data Plot of Vehicle_1 Straight Level Flight 
Table 1. List of Performance Parameters

\begin{tabular}{|c|c|c|c|c|}
\hline Vehicle & Test & Parameters & Indication & $\begin{array}{c}\text { Raw Data to } \\
\text { Process }\end{array}$ \\
\hline \multirow{6}{*}{$\begin{array}{l}\text { Vehicle_1, } \\
\text { Vehicle_2, } \\
\text { Vehicle_3 }\end{array}$} & \multirow{6}{*}{$\begin{array}{l}\text { Flight } \\
\text { Performance } \\
\text { (Straight } \\
\text { Flight) }\end{array}$} & 1. Min/Max Velocity & Min/Max Vel_x & Vel_x \\
\hline & & 2. Lift, $\mathrm{C}_{\mathrm{L}}$ & Mass x (Acc_z-g) & Pos_z \\
\hline & & 3. Power Efficiency & $\begin{array}{c}\text { Lift/Watt, } \\
\text { Vel_x/Watt, } \\
\text { Watt/Weight, } \\
\text { Weight/ } \\
\text { Area(Wing Loading) }\end{array}$ & $\begin{array}{c}\text { Pos_z, Amp, } \\
\text { Volt }\end{array}$ \\
\hline & & 4. Flapping Efficiency & $\begin{array}{c}\text { Lift/Hz, Vel_x/Hz, } \\
\text { AoA }\end{array}$ & Pos_z, Vel_x \\
\hline & & 5. Twisting Performance & Up/Down Twist Ang & Ang_z \\
\hline & & 6. Advance Ratio(J) & Vel_x/(2bФf) & Param 1, 4 \\
\hline \multirow{4}{*}{ Vehicle_2 } & \multirow{4}{*}{$\begin{array}{l}\text { Takeoff } \\
\text { /Landing }\end{array}$} & 1. $\mathrm{T} / \mathrm{L}$ Distance & Taxi Distance & Pos_x,y,z \\
\hline & & 2. T/L Speed & Vel at T/L Point & Vel_x,y,z \\
\hline & & 3. Taxi Performance & $\begin{array}{c}\text { Taxi Radius of } \\
\text { Gyration }\end{array}$ & Pos_x,y \\
\hline & & 4. Climb Rate & After Takeoff Point & Pos_x,y,z \\
\hline
\end{tabular}

Table 2. Performance Data of the Vehicles

\begin{tabular}{|c|c|c|c|c|c|c|c|c|c|}
\hline \multirow{2}{*}{ Vehicle } & $\begin{array}{l}\text { Flight } \\
\text { No. }\end{array}$ & $\begin{array}{c}\text { Flapping } \\
\text { Freq }\end{array}$ & Power & Vavg & AoA & Lift & $\mathrm{C}_{\mathrm{L}}$ & $\begin{array}{c}\text { Twisting } \\
\text { Angle }\end{array}$ & \multirow{2}{*}{$\begin{array}{c}\mathrm{J} \\
\text { (Advance } \\
\text { Ratio) }\end{array}$} \\
\hline & $\begin{array}{c}\text { Straight } \\
\text { Level }\end{array}$ & $\mathrm{Hz}$ & Watt & $\mathrm{m} / \mathrm{s}$ & deg & $\mathrm{N}$ & - & deg & \\
\hline \multirow{5}{*}{ Vehicle_1 } & $\# 1$ & 15.55 & 12.60 & 4.96 & 16.55 & 1.22 & 1.05 & 25.64 & 0.73 \\
\hline & $\# 2$ & 17.39 & 15.34 & 5.61 & 14.62 & 1.37 & 0.94 & 33.77 & 0.74 \\
\hline & $\# 3$ & 18.21 & 17.24 & 5.84 & 14.13 & 1.38 & 0.87 & 28.50 & 0.73 \\
\hline & $\# 4$ & 19.37 & 18.56 & 5.67 & 14.35 & 1.36 & 0.92 & 36.35 & 0.67 \\
\hline & $\# 5$ & 21.30 & 24.71 & 6.29 & 13.43 & 1.48 & 0.81 & 50.08 & 0.68 \\
\hline \multirow{5}{*}{ Vehicle_2 } & $\# 1$ & 11.43 & 22.18 & 5.99 & 18.77 & 1.93 & 1.17 & & 0.55 \\
\hline & $\# 2$ & 12.24 & 28.79 & 7.18 & 16.38 & 2.01 & 0.84 & 71.53 & 0.61 \\
\hline & $\# 3$ & 12.25 & 31.43 & 6.19 & 17.67 & 1.92 & 1.08 & 81.30 & 0.53 \\
\hline & $\# 4$ & 12.27 & 24.30 & 6.12 & 18.15 & 1.93 & 1.15 & & 0.52 \\
\hline & $\# 5$ & 12.76 & 28.89 & 6.40 & 16.90 & 1.99 & 1.05 & 85.15 & 0.52 \\
\hline \multirow{5}{*}{ Vehicle_3 } & $\# 1$ & 18.02 & 16.07 & 4.97 & 18.51 & 1.33 & 1.15 & 33.83 & 0.63 \\
\hline & $\# 2$ & 19.66 & 17.19 & 4.77 & 19.28 & 1.31 & 1.25 & 33.11 & 0.56 \\
\hline & \#3 & 20.00 & 18.33 & 4.36 & 20.73 & 1.16 & 1.30 & 32.41 & 0.50 \\
\hline & $\# 4$ & 22.17 & 20.82 & 4.99 & 21.51 & 1.17 & 1.02 & 27.73 & 0.52 \\
\hline & $\# 5$ & 22.22 & 20.11 & 4.80 & 20.42 & 1.23 & 1.14 & 29.13 & 0.49 \\
\hline
\end{tabular}


twisting angle was calculated from the angle between the vertical position of the rear spar axis and that of the front spar axis during the flapping stroke.

Another performance parameter that is of concern in this work is the advance ratio, which is defined in Equation 1. The magnitude of the advance ratio indicates the aerodynamic unsteadiness of the flapping motion (where larger values are found in quasi-steady regimes), and may provide a convenient reference point for the thrust development and flying efficiency of a vehicle [9]. In the equation, $\mathrm{V}, \mathrm{b}, \Phi$, and f denote forward flight speed, wing span, flapping angle, and flapping frequency, respectively.

$$
J=\frac{V}{\frac{1}{2} b \times 2 \Phi \times 2 f}
$$
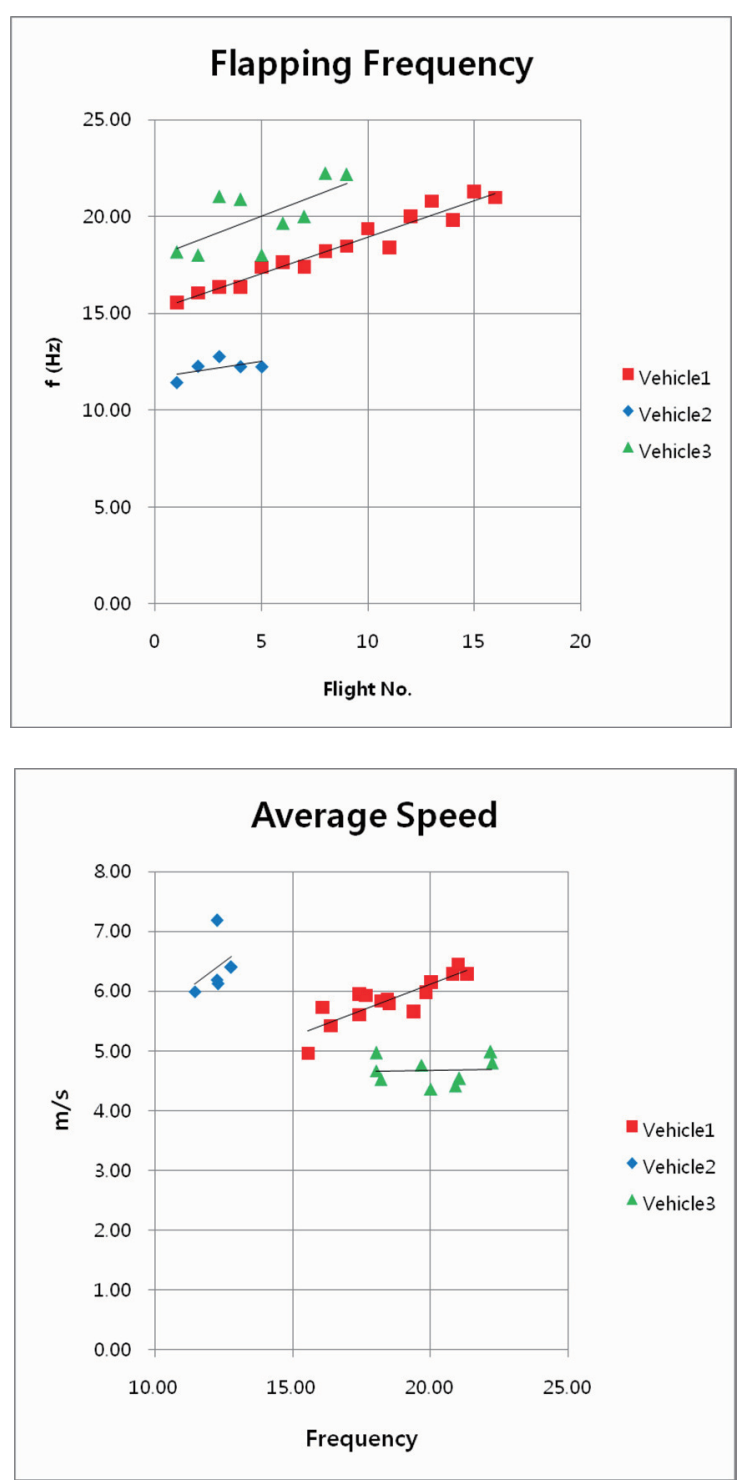

Some results of the performance parameters analysis are shown in Table 2. The range of flapping frequency as wide as possible was applied to each vehicle, considering the limits of the test space and the vehicle's flying capability. For example, the lower frequency range of vehicle_3 did not match that of vehicle_2 because vehicle_3 had insufficient power output. The results were divided into 4 categories for convenient assessment: overall flight performance, power efficiency, flapping efficiency, and takeoff/landing. Some twisting angle data are missing in the table because the tracking cameras were not able to follow the most dynamic flapping motion of vehicle_2 wings at every detecting frequency. Most of the data sets were completed by manually correlating the lost data with their addresses, but for some data addresses could not be located.
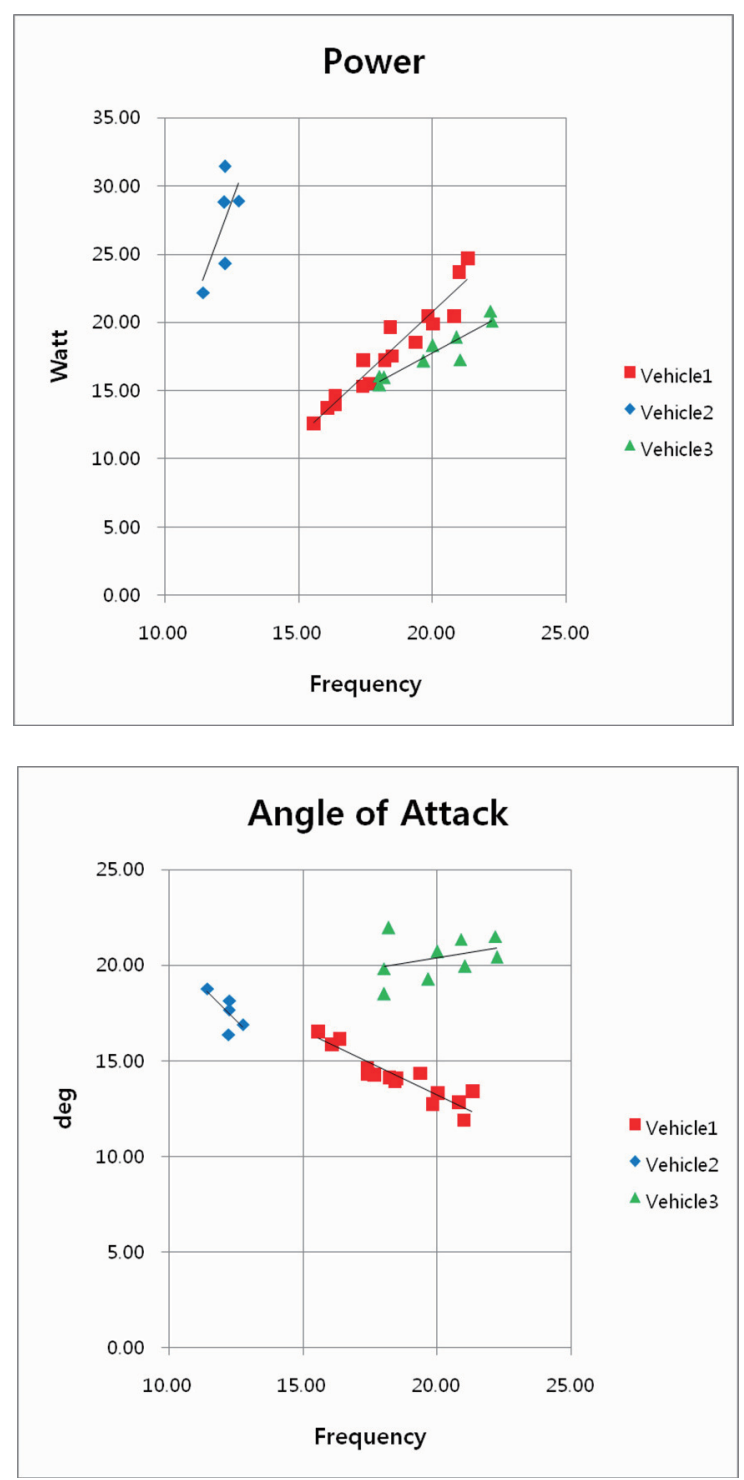

Fig. 11. Overall Flight Performance Plot of the Vehicles 
Fig. 11 illustrates the overall performance plots of the vehicles. Performance assessments inferred from the test results are summarized as follows:

(1) Vehicle_3 requires the fastest wing beating among the three vehicles even though the power it produced is not the lowest. It is presumed that vehicle_3's higher wing loading (than vehicle_1) induced this result, which is explained in detail in the following paragraph.

(2) Vehicle_2's high speed but low frequency is largely thought to be attributed to its active wing twisting, driven by the double driving crank mechanism. A much higher twisting angle in Table 2 supports this statement.

(3) Vehicle_3 shows a much larger AoA than vehicle_1 and vehicle_2 to maintain in-flight balance. From the vehicle weights and the graphs of AoA and average speed, it is inferred that a larger AoA is required to fly at slow speeds for light and small vehicles. This inference is clearer in the AoA graph: high vehicle speed utilizes a smaller AoA.

(4) Once the vehicles reach trim condition after release, their lift coefficients are not very different from each other due to the fact that they are under level flight. Their lift forces are roughly equivalent to their own weights, resulting in moderately-sized lift coefficients of around 1.0.

(5) Each vehicle's advance ratio is well below 1.0 (0.5 0.8), which indicates that their flights mostly rely on wing flapping rather than fixed-wing flight mechanisms [9]. It is presumed that the camber used in the wing's front spar of vehicle_1 contributes to the slightly higher J of vehicle_1 than those of the other vehicles, while the twisting effect of vehicle_2 wing compensates the J value again.

Fig. 12 illustrates the data plot of the vehicles related to power performance and efficiency. The following conclusions can be made:

(1) The graph of power per weight indicates that with respect to weight, the power efficiency of vehicle_1 or vehicle_3 is highest. However, this can be misleading: one must consider the fact that vehicle_1 and vehicle_3 have to operate with more wing beatings to get more power and maintain flight, while vehicle_2 still has room for more power (high speed flight tests were restrained for vehicle safety, considering the indoor space of the test chamber). Unlike the power efficiency investigation of fixed wing vehicles, the power efficiency investigation of a FMAV should include wing frequency, as shown in the second graph, to obtain a

Table 3. Wing Loadings of Vehicles in Level Flight

\begin{tabular}{|c|c|c|c|}
\hline \multirow{2}{*}{ Vehicles } & Weight & Wing Area & Wing Loading \\
\cline { 2 - 4 } & $\mathrm{G}$ & $\mathrm{m}^{2}$ & $\mathrm{~g} / \mathrm{m}^{2}$ \\
\hline Vehicle_1 & 130 & 0.0737 & 1,765 \\
\hline Vehicle_2 & 210 & 0.0749 & 2,805 \\
\hline Vehicle_3 & 120 & 0.0580 & 2,069 \\
\hline
\end{tabular}
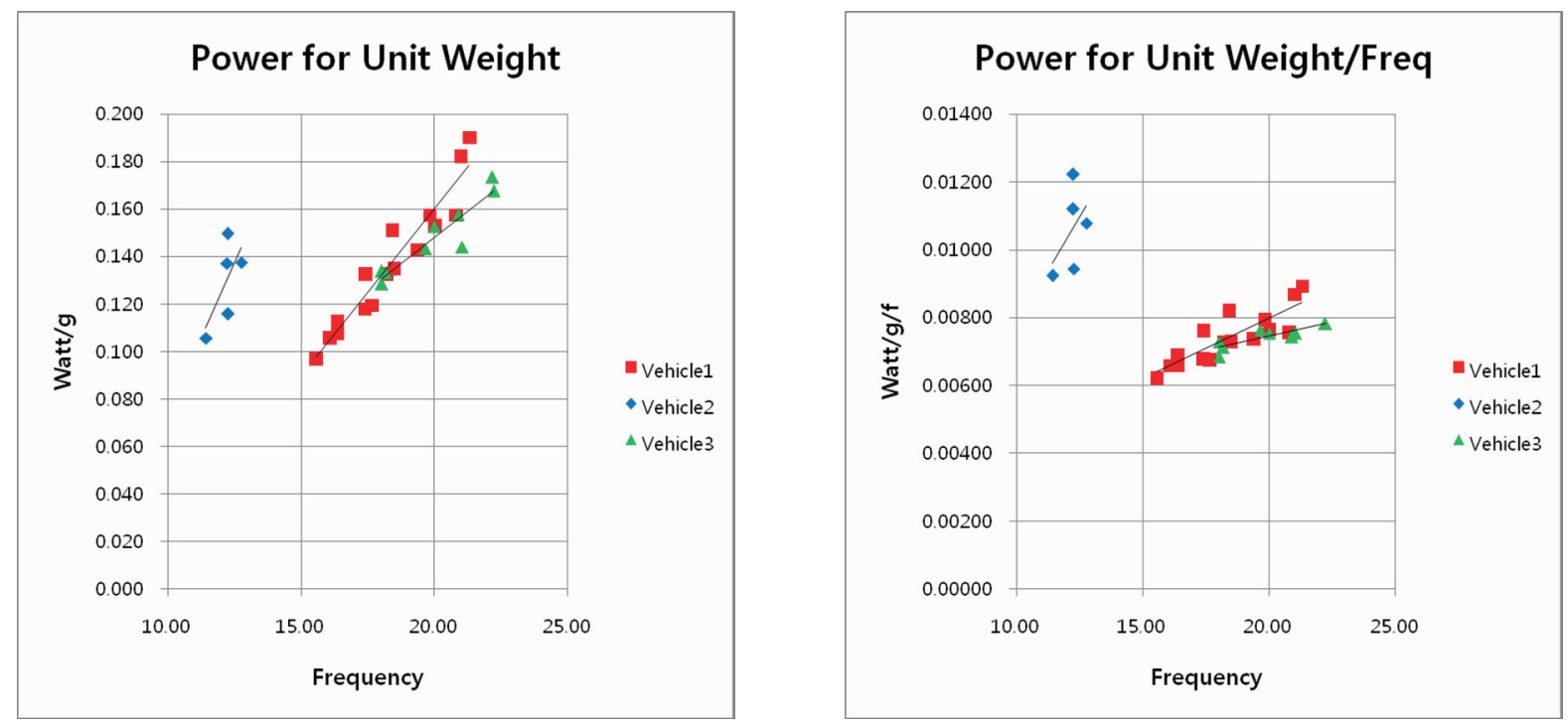

Fig. 12. Power Efficiency Plot of the Vehicles 
better overall perspective. From this standpoint, vehicle_2 has the largest power under the condition of equal weight and frequency.

(2) Vehicle_3's weight was originally $130 \mathrm{~g}$ to match vehicle_l's weight but vehicle 3 failed to fly. So, the battery of vehicle 3 was changed to reduce the weight of vehicle 3 by $10 \mathrm{~g}$. This suggests that the resulting data sets of vehicle_3 are those of a marginal flight between flyable and non-flyable. Much of this flying limitation is thought to originate from the small wing area and thus high wing loading, as shown in Table 3. The replacement of the wing with a wider one may solve the problem.

(3) The result that vehicle_2 displays the most notable performance in a straight-level flight test from the overall parameters assessment does not necessarily mean that it has the best flying ability. Because the straight-level test was the only test done in the chamber, additional performance assessments are required to consider agility and high maneuverability metrics for future work. Considering the fact that vehicles with a lower wing loading are usually advantageous to sudden reactions such as sharp turn for obstacle evasion, it would seem likely that vehicle_1 and vehicle_3 will show better performance in agile maneuver tests.

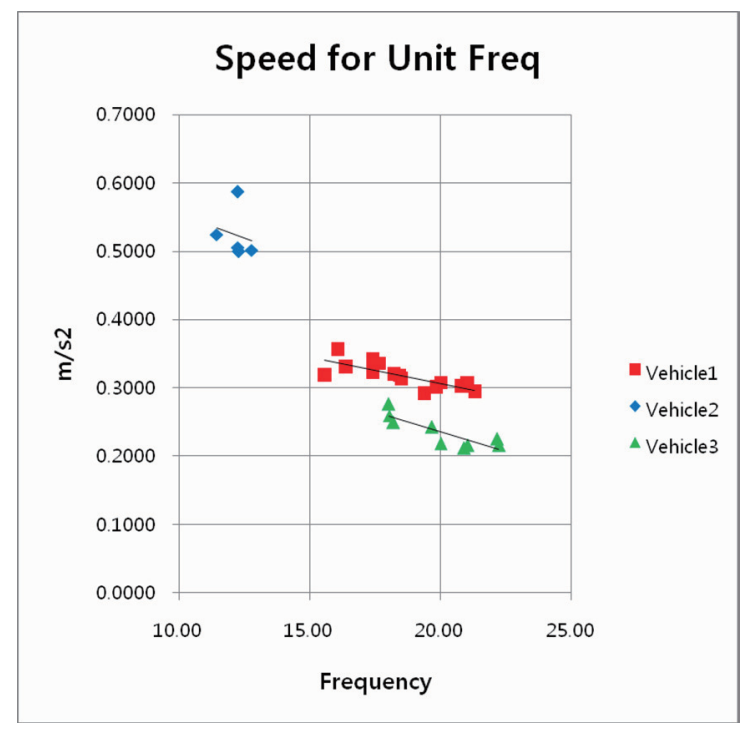

Fig. 13. Flapping Efficiency Plot of the Vehicles
Fig. 13 illustrates the data plot of average forward speed for a single wing beating. This plot can be used to assess flapping efficiency. Considering that a vehicle's power production is closely related to the vehicle's thrust and speed, it is obvious the speed per wing beating of vehicle_2 is highest.

Lastly, the Vicon data prove that the numerical measurement of a vehicle's takeoff and landing ability is enabled by associated performance parameters as shown in Table 4 . The resulting data are based on the smooth ground of the test chamber, so the data do not take various realistic terrain conditions into consideration, although they should be considered in future work.

While the takeoff distance denotes the straight taxiing distance from the departure to takeoff, taxi radius parameter is used for landing performance because the circular taxiing (not straight taxiing) was tested for the complete stop of the landing gear for the reason that the landing distance is sensitive to the ground condition. Therefore the taxi radius instead of distance is specified for the landing data.

For more extensive understanding of the flight characteristics of the FMAVs, computational fluid and structure analyses were also performed. Two-way FSI (FluidStructure Interaction) analysis was used in the research, and some of the results such as wing deflection and aerodynamic flow are displayed in Fig. 14 and 15. Analysis data showed good agreement with the test data. These analysis efforts were made to keep up with the prior studies about unsteady aerodynamic analyses of a flapping flight such as those in the reference $[10,11]$. Detailed analysis procedure and result will be reported in a separate paper.

\section{Conclusion}

A series of flight test measurements for three different FMAVs was conducted using a tracking system in an indoor MAV test facility, for numerical performance analysis and assessment of the vehicles. Practical test procedures and analysis methods were employed to obtain practical and useful results. The findings from the tests indicated that FMAV flight performance parameters can be measured for use in numerical performance evaluations. The strategies

Table 4. Takeoff/Landing Performance of Vehicle_2

\begin{tabular}{|c|c|c|c|c|}
\hline \multirow{2}{*}{ Vehicle_2 } & Distance & Speed & Taxi Radius & Climb Rate \\
\cline { 2 - 5 } & $\mathrm{m}$ & $\mathrm{m} / \mathrm{s}$ & $\mathrm{m}$ & $\mathrm{deg}$ \\
\hline Takeoff & 11.22 & 7.23 & N/A & 11.16 \\
\hline Landing & N/A & 5.53 & 1.04 & N/A \\
\hline
\end{tabular}


used here, and the resulting conclusions, will be beneficial for future systematic assessments of enhanced performance of a design-upgraded vehicle, for there are typically numerous prototype changes and improvements in FMAV development.

The present test metrics should be useful for the test and evaluation of general types of MAVs. Thus, this testing method will be useful for the development of future MAV system, as well.

\section{Acknowledgements}

This study was supported by Agency for Defense

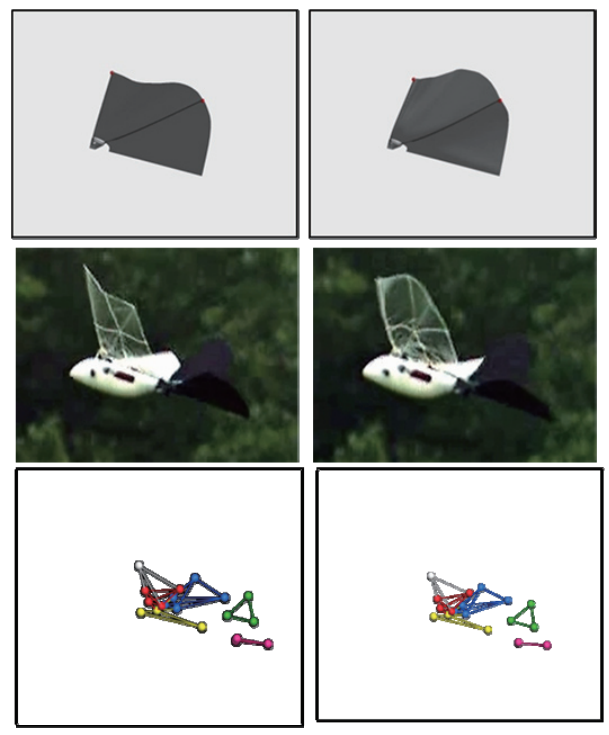

(a) Wing Deflection of Analysis, Camera, Vicon
Development (ADD) of Korea and Air Force Research Laboratory (AFRL) of USA under the Joint Research Project Agreement (RDT\&E: KS AF-10-0001): Analysis and Construction of Micro Air Vehicle.

\section{References}

[1] Shyy, W., Lian, Y., Tang, J., Liu, H., Trizila, P., Stanford, B., Bernal, L., Cesnik, C., Friedmann, P., Ifju, P., “Computational Aerodynamics of Low Reynolds Number Plunging, Pitching and Flexible Wings for MAV Applications", Acta Mechanica Sinica, Vol. 24, No. 4, 2008, pp. 351-373.

[2] Azuma, A., Masato, O., Kunio, Y., "Aerodynamic

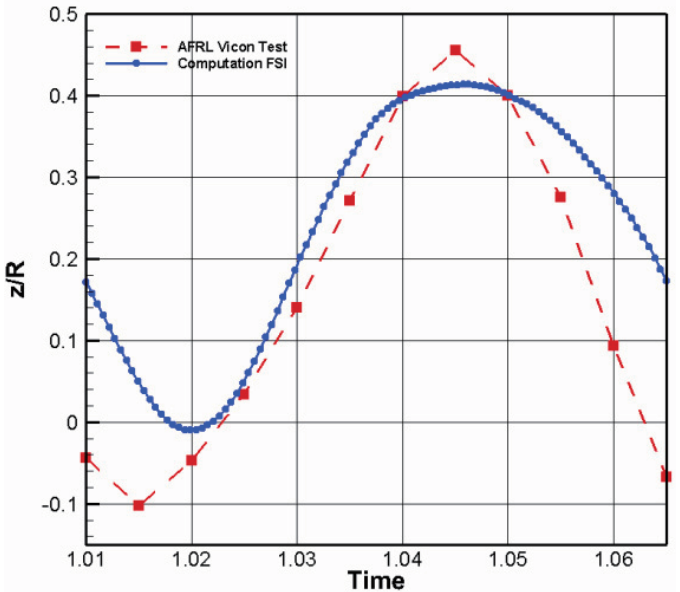

(b) Left Wing Tip Height per Half Span Length

Fig. 14. Wing Motion Comparison between FSI Analysis and Test

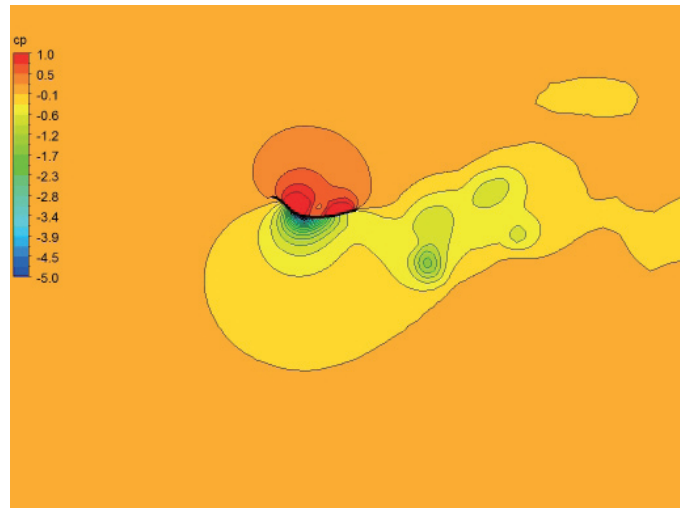

(a) Pressure Field during Upstroke

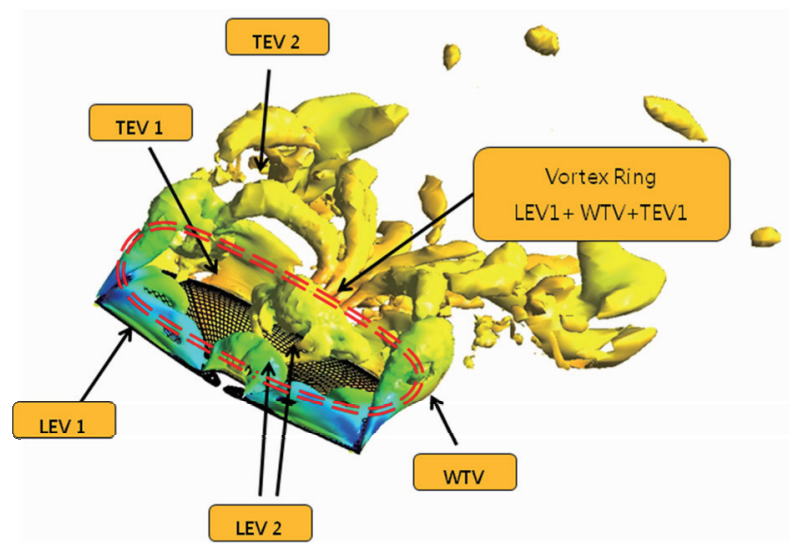

(b) 3-Dimensional Wing Vortex Structure

Fig. 15. Representation of Wing Flapping Principle by FSI Analysis 
Characteristic of Wings at Low Reynolds Numbers", Progress in Astro. \& Aero., Vol. 195, Mueller TJ (ed.), 2001, pp.341398.

[3] Vicon Motion Systems, http://www.vicon.com

[4] Ulrich, E., Humbert, J., Pines, D., "Pitch and Heave Control of Robotic Samara Micro Air Vehicles", Journal of Aircraft, Vol. 47, No. 4, 2010, pp. 1290-1299.

[5] Grauer, J., Ulrich, E., Hubbard Jr., J., Pines, D., Humbert, J., "System Identification of an Ornithopter Aerodynamics Model", AIAA Atmospheric Flight Mechanics Conference, Toronto, Canada, August 2010.

[6] Lin, C., Hwu, C., Young, W., “The Thrust and Lift of an Ornithopter's Membrane Wings with Simple Flapping Motion", Aerospace Science Technology, Vol. 10, No. 2, 2006, pp. 111-119.

[7] Stanford, B., Beran, P., and Kobayashi, M., "Aeroelastic
Optimization of Flapping Wing Venation: A Cellular Division Approach", AIAA Journal, accepted for publication, 2012.

[8] Warrick, D., Bundle, M., Dial, K., "Bird Maneuvering Flight: Blurred Bodies, Clear Head”, Integrative Comparative Biology", Vol. 42, No. 1, 2002, pp. 141-148.

[9] Ho, S., Nassef, H., Pornsinsirirak, N., Tai, Y., Ho, C., "Unsteady Aerodynamics and Flow Control for Flapping Wing Flyers”, Progress in Aerospace Sciences, Vol. 39, 2003, pp 635-681.

[10] Liu, H., "Integrated Modeling of Insect Flight: from Morphology, Kinematics to Aerodynamics", Journal of Computational Physics, Vol. 228, No. 2, 2009, pp. 439-459.

[11] Kim, J. H. and Kim. C., "Computational Investigation of Three-Dimensional Unsteady Flowfield Characteristics around Insects' Flapping Flight", AIAA Journal, Vol. 49, No. 5, 2011, pp. 953-968. 